\title{
Is there any Correlation between Pulse Rate and Ophidophobia?
}

\section{Muhammad Imran Qadir and Sabeeha Rana*}

Institute of molecular biology and biotechnology, Bahauddin Zakariya University, Multan, Pakistan

*Corresponding Author: Sabeeha Rana, Institute of molecular biology and biotechnology, Bahauddin Zakariya University, Multan, Pakistan.

Received: August 08, 2019; Published: September 04, 2019

DOI: 10.31080/ASMI.2019.02.0360

\section{Abstract}

Pulse rate is the speed of heartbeats, which is measure in one minute. It depends on the individual's age body weight and it is variable from person to person. Normal pulse rate of healthy person is 60 to 100 beats per minute. Elevated and reduced pulse rate may indicate some medical conditions. Ophidophobia is fear from every type of snakes it is also varies from person to person. Bad experience with snakes is the major cause of ophidophobia. Different types of treatments like cognitive therapy and medications are used. Study objective was to check the correlation between ophidophobia and pulse rate of individuals. Radial pulse method is use to measure the pulse rate. $P$ value of males and females considered as non-significant. There is no relation between ophidophobia and pulse rate of individuals.

Keywords: Pulse Rate; Ophidophobia; Snakes

\section{Introduction}

Pulse rate means the number of heartbeats per minute. It may vary from individual to individual. When a person is at resting condition then the pulse rate is lower and when a person is exercising then the pulse rate is higher because our body needed more oxygen [1]. Normal pulse rate of an adult is 60 to 100 beats per minute and for children 70 to 100 beats per minute. Factors that affect the pulse rate are age, temperature, body position, emotions, medication and body size. These factors are involve in increasing and decreasing the pulse rate [2]. There are different pulse sites where we can check or measure the pulse rate, that sites are temporal, carotid, brachial, radial, femoral, popliteal, dorsalis pedis. Elevated pulse rate gives the indication of heart related conditions such as high or low blood pressure level, thyroid disorder, stress, anxiety, shock, alcohol and anemia. Reduced pulse rate gives the indication of some type of infections, complications during heart surgery, some medications, hypothyroidism and inflammatory diseases such as rheumatic fever [3].

Ophidophobia is a phobia of snakes in which individuals are afraid of snakes and snake related things. Ophidophobia may vary from individual to individual. Mostly females and children are more victims of this snake phobia. Initially the individual is not aware of phobia but with the passage of time the individual know about that, he is afraid of snakes. There are many reasons behind ophi- dophobia [4]. One of the main reason is the bad experience with the snakes in the past. Some people are not only afraid of snakes but they are also afraid from their appearances because appearance of snakes is dangerous. Some snakes are not poisonous and dangerous but individuals who are suffering from ophidophobia are afraid of every type of snakes [5]. Ophidophobia may be mild or may be an extreme fear from snakes. Individuals limit themselves in the houses they do not go outside or the places where there may be a chance of appearing snakes. Symptoms of ophidophobia are shivering, panic attacks, dry mouth, increase heartbeat. Treatment for ophidophobia by different medications, exposure therapy and cognitive therapy [6].

\section{Objective of study}

Objective of study was to check the relation between pulse rate of individuals and Ophidophobia.

\section{Materials and Methods}

Firstly we selected the students randomly to check their pulse rate and we ask about ophidophobia either they have ophidophobia. We use radial pulse method to check their pulse rate.

Place the index finger and middle finger on the left arm just right below the thumb and gently press the fingers to find out the pulse. Once the pulse was recognized then use your clock with other hand 
in order to count the pulses. Look at the clock and count the number of heartbeats in 1 minute. To check the pulse by using thumb should be avoid because it causes hurdle in counting heartbeats.

\section{Results}

Most of the individuals said that they are afraid of snakes and some said that they are not. Some individuals have an extreme fear of snakes. Total 100 individuals of both male and female selected to check their pulse rate. In case of ophidophobia, average value and standard deviation of females are $76.62 \pm 10.99$ while average value and standard deviation of males are $80.5 \pm 9.03$ and average value and standard deviation of both males and females' are78.21 \pm 10.34 . In case of no ophidophobia, average value and standard deviation of females are $70.83 \pm 7.92$ while average value and standard deviation of males are $73.26 \pm 11.37$ and average value of both males and females are72.18 \pm 9.89 . After finding the mean and standard deviation, test was required to find the $\mathrm{p}$ value $\mathrm{P}<0.1$ considered the significant as $P=0.001$. $P$ value of males are $0.09, p$ value for females are 0.02 , and $p$ value for both males and females was 0.1 and these results considered non-significant. Therefore, the results shows that there is no relation between ophidophobia and pulse rate of individual.

\begin{tabular}{|c|c|c|c|}
\hline Gender & Ophidophobia & No ophidophobia & \\
\hline & Mean \pm SD & Mean \pm SD & P Value \\
\hline Female & $76.62 \pm 10.99$ & $70.83 \pm 7.92$ & 0.094 \\
\hline Male & $80.5 \pm 9.03$ & $73.26 \pm 11.37$ & 0.025 \\
\hline Both & $78.21 \pm 10.34$ & $72.18 \pm 9.89$ & 0.1 \\
\hline
\end{tabular}

Table 1: Relation of the pulse rate with Ophidophobia.

\section{Conclusion}

It was conclude from the results that there is no relation between ophidophobia and pule rate of individuals.

\section{Bibliography}

1. Bulte CS., et al. "Level of agreement between heart rate variability and pulse rate variability in healthy individuals". European Journal of Anaesthesiology (EJA) 28 (2011): 34-38.

2. Khandoker AH., et al. "Comparison of pulse rate variability with heart rate variability during obstructive sleep apnea". Medical engineering and physics 33 (2011): 204-209.

3. Hamer M., et al. "Pulse rate reactivity in childhood as a risk factor for adult hypertension: the 1970 British cohort study". Journal of hypertension 34 (2016): 1804-1807.
4. Polák J., et al. "Fear the serpent: A psychometric study of snake phobia". Psychiatry Research 242 (2016): 163-168.

5. Isbell LA. "Snakes as agents of evolutionary change in primate brains”. Journal of human evolution 51 (2006): 1-35.

6. Åhs F., et al. "Disentangling the web of fear: amygdala reactivity and functional connectivity in spider and snake phobia". Psychiatry Research: Neuroimaging 172 (2009): 103-108.

Volume 2 Issue 10 October 2019 (c) All rights arereserved by Muhammad Imran Qadir and Sabeeha Rana. 